\title{
Sleep, Sleep Disorders, and Mild Traumatic Brain Injury. What We Know and What We Need to Know: Findings from a National Working Group
}

\author{
Emerson M. Wickwire ${ }^{1,2} \cdot$ Scott G. Williams ${ }^{3,4}$ - Thomas Roth $^{5} \cdot$ Vincent F. Capaldi $^{6} \cdot$ Michael Jaffe $^{7,8,9}$. \\ Margaret Moline $^{10}$ • Gholam K. Motamedi ${ }^{11}$ - Gregory W. Morgan ${ }^{12}$ • Vincent Mysliwiec ${ }^{4,13}$ • \\ Anne Germain ${ }^{14}$ • Renee M. Pazdan ${ }^{15}$ • Reuven Ferziger ${ }^{16}$ • Thomas J. Balkin ${ }^{6}$ • Margaret E. MacDonald ${ }^{17}$. \\ Thomas A. Macek $^{18} \cdot$ Michael R. Yochelson $^{19,20} \cdot$ Steven M. Scharf ${ }^{2} \cdot$ Christopher J. Lettieri $^{4}$
}

Published online: 22 March 2016

(C) The American Society for Experimental NeuroTherapeutics, Inc. 2016 therapeutic interventions for mild TBI (mTBI). First, mTBI and sleep physiology are briefly reviewed. Next, essential empirical and clinical questions and knowledge gaps are addressed. Finally, actionable recommendations are offered to guide active and efficient collaboration between academic, industry, and governmental stakeholders.

Keywords Sleep $\cdot$ Sleep disorders · Traumatic brain injury · Concussion $\cdot$ Insomnia $\cdot$ Sleep apnea

Emerson M. Wickwire

ewickwire@medicine.umaryland.edu

1 Department of Psychiatry, University of Maryland School of Medicine, Baltimore, MD, USA

2 Sleep Disorders Center, Division of Pulmonary and Critical Care, Department of Medicine, University of Maryland School of Medicine, Baltimore, MD, USA

3 Department of Medicine, Walter Reed National Military Medical Center, Bethesda, MD, USA

4 Department of Medicine, Uniformed Services University of the Health Sciences, Bethesda, MD, USA

5 Sleep Disorders and Research Center, Henry Ford Hospital, Detroit, MI, USA

6 Department of Behavioral Biology, Walter Reed Army Institute of Research, Center for Military Psychiatry and Neuroscience Research, Silver Spring, MD, USA

7 Department of Neurology, University of Florida, Gainesville, FL, USA

8 Concussion and Sports Program, University of Florida Trauma, Gainesville, FL, USA
9 UF Health Sleep Disorders Center, Gainesville, FL, USA

10 Eisai, Inc., Woodcliff Lake, NJ, USA

11 Department of Neurology, Georgetown University Hospital, Washington, DC, USA

12 Sleep Disorders Center, National Intrepid Center of Excellence, Bethesda, MD, USA

13 Department of Sleep Medicine, San Antonio Military Medical Center, San Antonio, TX, USA

14 Department of Psychiatry, University of Pittsburgh School of Medicine, Pittsburgh, PA, USA

15 U.S. Public Health Service, Fort Carson, CO, USA

16 Merck and Co, Kenilworth, NJ, USA

17 Defense and Veterans Brain Injury Center, (Contractor, General Carson, CO, USA

18 Department of Clinical Science, CNS, Takeda Development CenterAmericas, Deerfield, IL, USA

19 Medstar National Rehabilitation Network, Washington, DC, USA

20 Departments of Neurology and Rehabilitation Medicine, Georgetown University School of Medicine, Washington, DC, USA Dynamics Health Solutions), Evans Army Community Hospital, Fort 


\section{Introduction}

By any measure, mild traumatic brain injury (mTBI) is a major public health concern in the USA and worldwide. According to the Centers for Disease Control and Prevention, approximately 1.7 million Americans sustain a TBI each year, with approximately $70 \%$ (i.e., 1.2 million) considered mild $[1,2]$. Others have noted that the actual number of TBIs annually is likely much higher, as many go undiagnosed, unreported, and thus uncounted [2-4]. Regardless, there is no question that TBI results in substantial costs at the individual and societal levels, with estimated total expenditures exceeding \$21.5 billion per annum for mTBI alone (\$16.7 in 2003 US dollars) [5].

Disturbed sleep is among the most common complaints of patients with $\mathrm{mTBI}$ and contributes to morbidity and longterm sequelae [6]. Sleep disturbances can develop acutely following injury or emerge later during recovery, at times persisting for years after the initial injury. Although the exact mechanisms through which TBI affects sleep and circadian health are not yet fully understood, it is clear that sleep disturbances and resultant sleep disorders can impair the brain injury recovery process. Consequences can include further neurodegeneration, as well as exacerbation of the many sequelae and comorbidities of mTBI, including fatigue, hypersomnolence, depressed mood, anxiety, post-traumatic stress symptoms or post-traumatic stress disorder (PTSD), chronic pain, cognitive and functional impairments, and diminished healthrelated quality of life (HRQoL). As a result, sleep and circadian disturbances represent a unique, novel, and modifiable treatment target that can potentially improve outcomes in patients with mTBI. Initial findings suggest that focused sleep interventions may not only improve sleep disturbances, but also improve both HRQoL and functional outcomes in patients with mTBI. However, some critical and basic questions remain regarding the relationship between mTBI and sleep and circadian health, as well as treatment of sleep and circadian disturbances in the context of mTBI. Addressing these questions will require a sustained, collaborative effort involving diverse areas of expertise and stakeholders, with the "payoff" of improved clinical care and outcomes in mTBI.

The purpose of the present paper is to present key findings from a national working group on sleep and $\mathrm{mTBI}$, with a specific focus on the testing and development of sleeprelated therapeutic interventions for mTBI. This group, which included leading experts from academia, government, and industry, was assembled in 2014 as part of the development of a joint meeting on "TBI Treatment Development" by the American Society for Experimental Neurotherapeutics (ASENT) and the International Society for CNS Clinical Trials and Methodology (ISCTM). Preliminary findings were presented at that meeting in Washington, DC, in February 2015. This Current Perspectives article refines and expands the initial findings of the working group, with particular emphasis on research and systems-level recommendations to advance rapidly the state of the science and increase access to care. First, a brief overview of mTBI and sleep is presented. Next, essential scientific questions and knowledge gaps are addressed. Finally, actionable recommendations are offered to guide active and efficient collaboration between academic, industry, and governmental stakeholders.

Based on an evaluation of the existing literature, the current understanding of the interaction between mTBI and disturbed sleep, and an assessment of future needs, the working group formulated the following 6 essential questions:

1. Why does sleep matter in mTBI?

2. How should we be measuring sleep in mTBI?

3. What sleep disorders occur following mTBI?

4. What are the potentially unique mechanisms underlying sleep disturbance in $\mathrm{mTBI}$ ?

5. What are the most promising sleep-related treatment development opportunities?

6. What are the critical methodological issues that must be considered when exploring the relationship between sleep and $\mathrm{mTBI}$ ?

\section{Clinical Overview}

\section{Mild TBI}

Broadly defined, TBI refers to a category of injury involving transient amnesia, an alteration in consciousness, and/or loss of consciousness resulting from any force applied to the head or body [7, 8]. Based on factors assessed at the time of injury [e.g., level of responsiveness (e.g., [9]), duration of loss of consciousness, and duration of post-traumatic amnesia], as well as findings on neuroimaging, TBIs are classified as mild (mTBI or concussion), moderate, or severe. Table 1 illustrates this classification system [10].

In patients with typical mTBI, standard acute structural imaging studies such as head computed tomography scans do not show hemorrhage or other overt structural abnormalities. Specialized imaging modalities with magnetic resonance imaging, such as susceptibility weighted imaging, may show evidence of old hemosiderin as a proxy for diffuse axonal injury, and newer imaging techniques such as diffusion tensor imaging and functional magnetic resonance imaging hold promise for future clinical use [11]. However, an absence of findings, even on highly specialized imaging modalities, does not rule out mTBI, as the neurological sequelae of mTBI are believed to be caused by a transient disruption of brain function, including impairment of synaptic transmission, alterations in glucose metabolism, changes in cerebral blood flow, 
Table 1 Classification of traumatic brain injury

\begin{tabular}{lll}
\hline Mild ("concussion") & Moderate & Severe \\
\hline Normal imaging & Normal or abnormal imaging & Abnormal imaging \\
Loss of consciousness (LOC): 0-30 min & LOC $>30 \mathrm{~min}<24 \mathrm{~h}$ & LOC $>24 \mathrm{~h}$ \\
Alteration of consciousness (AOC): up to 24 h & AOC $>24 \mathrm{~h}$ & \\
Post-traumatic amnesia (PTA): 0-1 day & PTA $>1$ and $<7$ days & PTA $>7$ days \\
\hline
\end{tabular}

and impaired axonal function [12]. Further complicating the lack of objective structural pathology evident when standard clinical imaging techniques are employed, mTBI is a remarkably heterogeneous condition with varying degrees of clinical manifestations and severity. In addition, patient-level characteristics such as age, sex, premorbid headaches, and psychiatric conditions likely influence resilience and vulnerability to mTBI sequelae, as well as persistence of postconcussive symptoms. Importantly, while insufficient and disturbed sleep impair neurocognitive performance and increase accident risk, and thus likely increase risk for TBI events, little is known about how premorbid sleep disturbances affect recovery and long-term TBI sequelae.

As a result of this nonspecificity, consequences of mTBI are typically classified into various symptom clusters. Although there is a lack of consensus regarding symptom classification, one commonly used system of nomenclature divides symptoms into 3 clusters: physical (i.e., headaches, dizziness, fatigue, disequilibrium, photophobia, sleep disturbances), cognitive (i.e., difficulties with attention and concentration), and emotional domains (i.e., irritability, depression, anxiety) [10]. Notably, the Centers for Disease Control mTBI Assessment Classification system recognizes that sleep disturbances can affect clinical assessments following injury and emphasizes the importance of sleep disturbances in these individuals. In this classification scheme, disturbed sleep is incorporated as its own independent category, resulting in 4 symptom clusters: sleep, physical, cognitive, and emotional [13]. Of course, there is a great deal of overlap between these symptom clusters, with bidirectional interactions making it difficult to discern causality (e.g., disturbed sleep can both cause and be caused by headaches and irritability). Importantly, the centrality of sleep to mTBI is further evidenced in recovery, with sleep-related processes being increasingly implicated in the neuroplastic recovery from postmTBI symptoms [14].

\section{Sleep Physiology}

All animal species are known to have daily periods of inactivity and perceptual disengagement from the environment, known as sleep. In virtually all mammalian species, sleep is composed of 2 distinct states. These are rapid eye movement (REM; in adult humans about $20 \%$ of the night) and nonREM (NREM; in adult humans about $80 \%$ of the night) sleep.
These states differ from each other as much as they individually differ from wakefulness. NREM sleep is characterized by a progressive synchronization of electroencephalography (EEG) activity (NREM stages 1, 2, and 3) in parallel with an increase in arousal threshold. In contrast, REM sleep is characterized by EEG activation, rapid eye movements, and skeletal muscle atonia. These distinct states are hypothesized to perform independent and complementary physiologic and mental restorative functions [15]. Standardized criteria for defining different stages in humans have been published elsewhere [16].

Most sleep-related physiological functions, including the regulation of sleep and wakefulness, are controlled by 2 processes: a homeostatic process (process $\mathrm{S}$ ) and a timing process (process $\mathrm{C}$ ). Process $\mathrm{S}$ is a homeostatic input, or sleep drive, determined by the duration of prior sleep and wakefulness, and, at least in part, thought to be mediated by the extracellular biochemical substrate adenosine. Simply, levels of adenosine, and, consequently, a greater drive for sleep, progressively increase with prolonged wakefulness, which increase sleep propensity. Process C is modulated by circadian timing, which determines rhythmic variations of sleep and wakefulness over the 24-h period. This process is generated by the suprachiasmatic nuclei and modulated by melatonin secreted by the pineal gland, which is itself regulated by exposure to ambient levels of blue light. While numerous physiologic processes are regulated by process $\mathrm{C}$, core body temperature and dim light melatonin onset are the most commonly used indicators of the phase of the circadian pacemaker. The interaction between process $\mathrm{S}$ and process $\mathrm{C}$ determines the propensity for sleep (or wake) across the 24-h day. As will be discussed, alterations in homeostatic sleep drive and circadian rhythmicity have direct relevance for sleep-wake disturbances following mTBI.

The switching between sleep and wake is complex and involves the interaction of numerous neural centers in the brain, including several hypothalamic and brainstem nuclei. Principal centers include the ventrolateral preoptic nucleus, which contains $\gamma$-aminobutyric acid and galanin producing neurons that, when stimulated, are responsible for normal sleep. The posterior lateral hypothalamus contains orexinproducing hypocretin neurons, which are responsible for wakefulness. In addition to orexin, histamine (released from the tuberomammillary nucleus), serotonin (dorsal raphe 
nucleus), and noradreneline (locus coeruleus) are other important neurotransmitters for maintaining wakefulness. These sleep and waking functions are mutually exclusive opponent processes, such that promoting sleep inhibits wake and promoting wake inhibits sleep. Similar "switches" regulate the transitions between NREM and REM sleep [17]. With regard to sleep disturbances in mTBI, any imbalance in these neurotransmitter relationships, or damage to these structures or the connections between them, can lead to alterations in normal sleep or wakefulness. Injuries to these neural structures or impairments in related brain functions can result from mTBI, causing sleep-wake disturbances.

Disturbances of sleep can be classified into 4 categories, each of which has been reported in patients with mTBI: insufficient sleep duration or continuity, alterations in sleep stages (i.e., changes in sleep architecture), pathologies in sleep (sleep disorders), and abnormalities of the timing of sleep (circadian rhythm disorders). The daytime consequences of such sleep and circadian disturbances include impairments in psychomotor performance; cognitive function, including moral judgment; mood; and endocrine, metabolic, immune, inflammatory, and cardiovascular function [18, 19]. These deficits occur following experimentally induced acute sleep disruption and chronic sleep disruption, as well as in clinical samples.

Although a unifying theory that definitively explains the purpose of sleep remains elusive, several theories have been proposed to explain its myriad functions [15]. For example, it has been postulated that sleep is required for restorative and somatic growth, a theory supported by the linkage of certain anabolic processes and hormone function such as growth hormone, which is secreted during deep NREM sleep. Perhaps most relevant to mTBI, recent evidence demonstrates the importance of sleep for neural growth and plasticity [20]. Indeed, sleep is central to learning and memory consolidation, brain development and restoration, and neuronal synaptic plasticity by eliminating weak connections and preserving strong connections within the synapses. A landmark study conducted by Xie et al. [21] demonstrated that sleep is associated with a marked increase in interstitial space associated with increased $\beta$-amyloid clearance in mice. Thus, sleep likely has a unique and neuroprotective role through the elimination of neurotoxic waste products that does not occur during wakefulness.

\section{Essential Questions Related to mTBI and Sleep}

\section{Why Does Sleep Matter in mTBI? Evidence from Animal Studies}

Poor subjective sleep quality is one of the most common complaints of patients who have experienced mTBI, with milder injury being associated with greater impairments in both subjective and objective measures of sleep [22]. Furthermore, the incidence of sleep disorder diagnoses is greater in patients with mTBI than in the general population. A recent metaanalysis of patients with TBI of mixed severity revealed that $50 \%$ of patients reported sleep complaints and approximately $30 \%$ were diagnosed with a clinical sleep disorder [6]. Specifically, patients with TBI experienced high rates of insomnia (29\%), hypersomnia (28\%), and sleep apnea (25\%). Unfortunately, comorbid sleep disturbance has often been viewed as a symptom of mTBI, rather than as a modifiable factor that can potentially affect the rate and extent of recovery. In reality, many postconcussive symptoms, such as anxiety, headaches, fatigue, and irritability, can result from, and exacerbate, sleep disturbances in a "vicious cycle".

In animal studies, evidence suggests that the effects of disturbed sleep and sleep loss on resilience to mTBI events depend, at least in part, upon the temporal relationship between the brain injury and sleep. Specifically, sleep deprivation in rodents may have salutary effects immediately prior to injury but negative effects immediately following injury [23-25]. In rats, Hsu et al. [24] observed that 5 days of total sleep deprivation prior to injury conferred a measure of protection against ischemic injury. Similarly, Moldovan et al. [25] found that a habitual decrease in total sleep time prior to TBI reduced the severity of injury. Although the physiological mechanisms underlying this protective effect are as yet unknown, there are several intriguing possibilities. First, it has been hypothesized that sleep deprivation prior to brain injury results in a form of "ischemic preconditioning", effectively habituating the brain to subthreshold noxious byproducts of cellular injury [26]. Similarly, an insufficient quantity or quality of sleep results in elevated levels of extracellular adenosine. Because adenosine is potentially neuroprotective, it could be hypothesized that both acute sleep loss and chronic sleep debt-induced elevations of extracellular adenosine help protect against TBI sequelae. Another possibility is that sleep deprivation prior to ischemic injury may alter the pattern of gene activation/ deactivation in a manner that affords some degree of neuroprotection [27]. Finally, sleep loss prior to injury may elicit rebound sleep following injury, thereby facilitating sleepenhanced neuronal regeneration and recovery [28]. It is worth reinforcing that the neuroprotective potential of preinjury sleep debt or its influence on subsequent outcomes has yet to be determined in humans. Prospective and longitudinal human studies are clearly required in order to determine the extent to which habitual sleep duration and/or sleep quality affects recovery from concussive events.

In contrast to the potentially protective effects of acute, preinjury sleep loss in rats, there is significant controversy regarding the impact of sleep loss on outcomes following mTBI. Several investigators have found that insufficient sleep 
postinjury may exacerbate brain injuries and impair long-term recovery. For example, Gao et al. [29, 30] found that 3 days of sleep deprivation and sleep disruption exacerbated injury patterns and impaired recovery in rats after simulated stroke. Similarly, sleep deprivation has been associated with a number of potentially relevant deleterious effects on brain homeostasis, including changes in brain temperature [31], glutamate concentrations [32], and energy consumption as evidenced by decreased levels of brain glycogen [33]. Sleep deprivation also results in elevated proinflammatory cytokines (interleukin- $1 \beta$, interleukin- 6 , and tumor necrosis factor- $\alpha$ ), which can impair neuronal growth and delay recovery $[34,35]$. If sleep loss incurs these neurophysiologic costs and also impairs neurorecovery, it is reasonable to hypothesize that postinjury sleep enhancement may facilitate neurorecovery. This hypothesis was explored in a study by Hodor et al. [36], who found that postinjury enhancement of NREM sleep with baclofen improved recovery from ischemic brain injury, as evidenced by increased neuronal sprouting and enhanced neurogenesis in the peri-infarct region of rats. Nevertheless, despite this promising finding, there remains a lack of clarity regarding the potential benefits of postinjury sleep. For example, Martinez-Vargas et al. [37] have shown that $24 \mathrm{~h}$ of total sleep deprivation after TBI decreased morphological damage and enhanced recovery in a rat model. Similarly, it has also been observed that hippocampal neurogenesis was enhanced in rats as a result of short-term sleep deprivation following injury [38].

It is important to realize that although fascinating and potentially impactful, the data discussed above are gained from animal studies, and the direct applicability to humans is unknown. Indeed, these studies highlight the challenging nature of translational science in mTBI. For example, the applicability to humans of these rodent brain injury models remains unknown, and preinjury sleep duration is a poor treatment target, owing to the unpredictable nature of mTBI. Nonetheless, these findings from animal studies provide valuable insight and suggest future directions for research in humans. Most obviously, the effects of sleep extension and sleep deprivation in various stages of injury warrant further exploration, as current clinical guidelines are lacking. For example, do patients with mTBI nap immediately or stay awake all night? In addition, there is a dramatic need for greater understanding of the neuroprotective and neurorecuperative mechanisms of sleep in mTBI, including exploration of the roles of specific sleep stages, neural substances, such as adenosine, and gene activation/ deactivation. Inflammatory processes similarly require further study and represent potential treatment targets in humans. In order to build on the exciting findings among animals, additional studies in humans are clearly needed. Our working group is presently exploring these translational issues.

\section{How Should we be Measuring Sleep in mTBI in Research Studies?}

To date, research studies have utilized inconsistent operational definitions regarding sleep and circadian patterns and disorders. Although physiologic sleep is defined by polysomnography (PSG) incorporating EEG and measures of muscle tone by electromyogram and eye movements by electrooculogram, overnight PSG is not always feasible or necessary to address the research question. Therefore, investigators have employed patient self-report, clinician rating, and the objective proxy of actigraphy to quantify sleep in patients with mTBI. Each of these approaches has strengths and weaknesses. The optimal sleep measurement research strategy depends on the primary question of interest and may combine more than one method for a given set of aims and hypotheses.

Certainly, thorough assessment of the multiple factors influencing sleep and mTBI is essential. In addition to sleep history and current sleep patterns, research assessment should include both the mechanism and severity of injury and other confounding factors, such as chronic pain or psychiatric disorders and medication use commonly associated with persistent postconcussive symptoms.

\section{Self-report Questionnaires}

Various self-report instruments have been used in studies of adult and pediatric patients with TBI [39-41]. To date, only the Pittsburgh Sleep Quality Index and the Epworth Sleepiness Scale have been validated against objective measures of sleep in patients with TBI [42-46]. Fichtenberg et al. [46] found that a cut-off of 8 of the global Pittsburgh Sleep Quality Index score, rather than the usual cut-off of 5 for nonconcussed samples, better discriminated between patients with and without clinically significant insomnia.

There are inherent limitations to self-report questionnaires [47]. Subjective measures of sleep quality and daytime functioning reflect the patient's experience of sleep, which are essential for the diagnosis of sleep disorders and evaluation of treatments. However, self-reported sleep does not always capture the nature or severity of underlying organic sleep pathology or clinical sleep disorder. Further, self-report can be compromised based on injury-related cognitive impairment, recency or salience effects (i.e., remembering only the most recent or unusual experiences), or reliance on data from indirect sources such as caregivers or parents of children [40]. For example, patients in hospitals or institutionalized settings (e.g., following polytrauma) have sleep-wake schedules and daily routines imposed upon them and are also frequently disrupted by medical practitioners and monitoring, which may mask existing sleep problems or contribute to the development of new sleep difficulties [47]. 


\section{Sleep Diaries}

Standardized sleep diaries are considered an essential measure of self-reported sleep patterns in both clinical and research settings. Sleep diaries have been used to demonstrate sleep disturbances in patients with mTBI relative to matched controls [47], as well as to measure treatment response to sleep treatments such as cognitive behavioral treatment for insomnia (CBTI) $[48,49]$. Sleep diaries offer the advantage of prospectively capturing sleep-wake patterns and behaviors that may interfere with or promote sleep, including the regularity of bed times and rise times, the frequency, timing, and duration of daytime naps, use of prescribed or nonprescribed stimulants (e.g., energy drinks, caffeine) or central nervous system depressants, including alcohol, and other relevant behaviors. The prospective collection of sleep data with the sleep diary, then, minimizes biases due to recency or salience effects. Furthermore, the sleep diary allows the patient or their caregiver to capture behaviors that may further exacerbate sleep and circadian disturbances, such as parasomnias. In the treatment of sleep disorders, sleep diaries facilitate longitudinal assessments to quantify adherence and progress over the course of treatment. Notably, there is a paucity of data regarding the psychometric properties of sleep diaries in patients with $\mathrm{mTBI}$, and there are few, if any, studies comparing sleep diary to PSG in this population. Fatigue and cognitive impairments may interfere with the ability of adult patients with mTBI to complete a sleep diary, and pediatric patients are often limited in their ability to self-monitor sleep. Although corroboration by bed partners, caregivers, or parents may improve accuracy, these individuals do not typically monitor the patient throughout their entire sleep period and therefore cannot provide an accurate accounting of quantity or quality of sleep [50]. Although patients with mTBI are not typically hospitalized, similar limitations are evident when considering reports of hospital staff, who typically only visit patients once per hour [51]. Despite these limitations, sleep diaries offer a low-cost, low-burden measure that can augment multimethod assessment of sleep and circadian patterns in patients with mTBI.

\section{Actigraphy}

An actigraph is a small, portable device typically worn on the nondominant wrist to monitor physical activity via an accelerometer. This ambulatory movement is scored via an algorithm to estimate sleep timing and duration (based on periods of inactivity, as defined by a pre-set threshold). These estimates can also be further manually edited based on sleep diary data to refine estimates of sleep and wakefulness patterns. Wrist actigraphy has been validated as a reliable proxy for sleep and circadian patterns in healthy adults and multiple sleep-disordered populations [51,52], and is frequently used as an outcome measure in sleep-related clinical trials. Importantly, wrist actigraphy can aid in the diagnosis of circadian rhythm disorders and evaluation of sleep in individuals unable to undergo PSG. Actigraphy presents many benefits and has been suggested as an ideal methodology for monitoring patients following brain injury [48]. Indeed, actigraphy has been used to study adult and adolescent patients with mTBI [41, 51, 53-58]. Like sleep diaries, actigraphy offers a low-burden method to estimate prospectively regularity, timing, and duration of sleep-wake patterns. Actigraphy can also be used in clinical trials over several weeks, enabling assessment of not only sleep-wake, but also 24-h physical activity and circadian rhythmicity [58]. Finally, in our clinical experience, actigraphy might be initially more acceptable than PSG to patients with mTBI, who can be irritable or agitated.

The use of actigraphy in mTBI samples may pose unique challenges. For example, the cost can be prohibitive for widespread use, and the validity of actigraphy in children and adults with mTBI is unknown. Further, low participation and early termination of actigraphic monitoring has been reported in adolescents with $\mathrm{mTBI}$, along with reports of failure to return the devices among both adolescents and adults [41, 51]. Similarly, despite scheduled reminders, Sinclair et al. [57] excluded $28 \%$ of enrolled participants from analysis, owing to prolonged noncompliance with actigraphy procedures. These authors also found moderate-to-strong associations between sleep diary reports and actigraphic assessment of sleep duration among both patients with TBI and matched healthy controls [57]. However, the agreement between these 2 methods weakened in patients with TBI, with sleep diary data of patients with TBI underestimating wake after sleep onset (WASO) and overestimating sleep onset latency (SOL). In patients with motor impairment, actigraphy tends to overestimate total sleep time [59]. Finally, studies to date have employed small sample sizes, primarily assessing mTBI, with limited data in moderate-to-severe TBI.

\section{$P S G$}

PSG is considered the standard measurement of sleep. The majority of studies employing PSG in patients with mTBI have reported alterations in sleep continuity and sleep stage architecture. Similarly, studies employing power spectral analysis have also identified EEG differences between patients with $\mathrm{mTBI}$ and matched healthy controls $[60,61]$. In spite of its clinical utility, PSG presents limitations. PSG is labor intensive and costly, and usually takes place in a sleep laboratory, a novel environment that may not capture a typical sleep pattern [62]. Even PSG performed in home settings involves monitoring devices that could interfere with normal sleep. Further, PSG recordings are generally time-limited, meaning that sleep cannot be recorded over multiple sleep periods, and are not suited for assessing circadian rhythms. Finally, PSG 
measurement of sleep and wake features reflect the summation of subcortical and cortical potentials from only a few electrodes on the scalp. Hence, the spatial resolution is greatly limited, and abnormal sleep processes or features that arise from specific brain regions may not be captured by PSG. In spite of these challenges, a recent study found 1-night PSG to be highly reliable for detecting obstructive sleep apnea (OSA) in patients with TBI of varying severity [63]. In addition to night-time measurement of sleep, daytime PSG, such as the multiple sleep latency test (MSLT), during which participants undergo a series of 4-5 timed daytime naps, can also be employed to measure daytime sleepiness. Objective evidence of daytime somnolence is required for diagnoses, including post-traumatic hypersomnia (mean MSLT $<8 \mathrm{~min}$ ) and post-traumatic narcolepsy (mean MSLT $<8$ min with the occurrence of 2 or more sleep-onset REM periods during the MSLT). In summary, PSG is a vital tool for evaluating sleepdisordered breathing, hypersomnia and parasomnias in patients with mTBI. In addition, it enables assessment of sleep architecture, a necessary but insufficient tool for understanding sleep-specific neurophysiologic aspects of mTBI.

\section{Advanced Sleep Measurement Methods}

Over the last 2 decades, the use of high-density EEG and sleep neuroimaging methods has steadily increased in both healthy and clinical populations [64-67]. Both approaches have further refined our understanding of the localized and neural underpinnings of healthy and disrupted sleep, as well as of circadian variations in brain functions [68-70]. The use of neuroimaging methods such as positron emission tomography and structural and functional magnetic resonance imaging during sleep and wakefulness in concussed, recovered, and never concussed individuals remain scarce, but has a high potential to provide unique and novel insights into the underpinnings of sleep and circadian disturbances in mTBI, and therefore may guide the development of innovative and targeted diagnostic and therapeutic strategies [70-75].

\section{What Sleep Disorders Occur Following mTBI?}

Subjective complaints of sleep-wake disturbances occur in $30-70 \%$ of those with TBI of all severity [76-80]. Insomnia, fatigue, and somnolence are the most common clinical diagnoses reported following mTBI, but sleep-disordered breathing can also develop. Parasomnias may also be a significant problem post-mTBI, but further study is needed to explore true prevalence and ascertain whether the apparent incidence is associated with use of selective serotonin reuptake inhibitor medications rather than a true sequela of mTBI.

The most common objective PSG findings in patients with mTBI are consistent with subjective reports of poor sleep quality. More specifically, patients with mTBI demonstrate increased sleep fragmentation, delayed sleep onset, increased awakenings and time awake during the night, and reduced sleep efficiency (e.g., [81]). Further, at least 3 studies have found that relative to matched healthy controls, patients with mixed-severity TBI demonstrate increased slow-wave sleep (SWS) [22, 82], although another study found reduced SWS and lower NREM delta power [83]. In light of the regenerative effects of SWS (e.g., axonal sprouting and synaptic remodeling), these findings appear to suggest a potential role for SWS in recovery from TBI. Further supporting this hypothesis, Sommerauer et al. [81] found that patients with TBI slept $2.5 \mathrm{~h}$ longer per 24 -h period-and also underestimated their sleep need-relative to matched healthy controls. Alterations in REM sleep have also been detected, including decrease in total REM sleep and reduced REM latency [77, 84, 85].

\section{Hypersomnolence}

Hypersomnia is a common and potentially very disabling consequence of TBI of mixed severity, including mTBI. Indeed, subjective complaints of daytime sleepiness are reported in $50-85 \%$ of individuals following head injuries [76, 77]. Both blast and blunt trauma head injuries can damage essential neural wakefulness circuits, including orexin- (hypocretin) producing cells in the hypothalamus leading to a centrally mediated hypersomnia [86]. Daytime sleepiness remits over time in some patients with TBI; however, this symptom persists in $10-53 \%$ [39, 54, 76-78, 80, 87]. It is not clear why some patients continue to experience clinically significant sleepiness, but both post-traumatic hypersomnia and posttraumatic narcolepsy are known to emerge in a subset of patients with mTBI. Similar to narcolepsy type 1, cerebrospinal fluid (CSF) hypocretin can be low or undetectable in these conditions if the head injury resulted in damage to the hypocretin-producing cells of the hypothalamus [54, 87]. In contrast, CSF hypocretin is typically normal in patients with narcolepsy type 2 (narcolepsy due to a medical condition) and other causes of central hypersomnia.

Treatment of daytime sleepiness can prove challenging in patients with mTBI as physical injuries, depression, and PTSD are common and can exacerbate or confound symptom presentation. Although it is generally recommended to restore the sleep-wake cycle and optimize treatment of comorbid diagnoses, there are currently no medications specifically approved for mTBI-related hypersomnia. Modafinil, armodafinil, methylphenidate, and amphetamines are often prescribed off-label for the treatment of daytime sleepiness. Notably, there is a paucity of data regarding the safety and efficacy of these medications among patients with mTBI. In a prospective, double-blind, randomized, placebo-controlled trial, Kaiser et al. [88] found that modafinil significantly 
improved both subjective and objective measures of sleepiness in patients with TBI. However, it did not reduce subjective fatigue, highlighting the multifactorial nature of daytime sequalae in this population. Similarly, Menn et al. [89] found that armodafinil improved sleep latency on MSLT despite relatively minor improvements in subjective sleepiness. Nonpharmacologic treatments have also been studied. Although not addressing sleepiness per se, Ponsford et al. [90] found that morning bright light therapy improved general fatigue in patients with mTBI.

Obstructive sleep apnea occurs at substantially higher rates in patients with TBI than the general population. Studies have reported OSA in $35-61 \%$ of patients with TBI $[44,76]$. In addition, patients with TBI with comorbid OSA experience greater neurocognitive impairments in memory and attention than those with similar TBI severity who do not have OSA [91]. Treatment is analogous to those with other causes of sleep-disordered breathing, where positive airway pressure (PAP) is the standard of care. However, the impact of therapy on neurocognitive recovery in patients with mTBI has not been studied. That said, treatment of OSA improves cognitive and executive functioning in patients without a TBI, although resolution of deficits and normalization of function does not occur in all individuals. It is thus unknown to what degree treatment of sleep-disordered breathing could improve function in patients with mTBI and OSA. Importantly, the benefit of OSA treatment is highly influenced by the type of therapy and therapeutic adherence. For example, although PAP is a highly effective treatment when used properly, many patients struggle to adhere to the therapy, limiting its real-world effectiveness [92]. This is especially concerning in patients with mTBI who might have insomnia or physical injuries that limit their ability to properly don and wear the device [93]. In addition, PTSD is common among patients with $\mathrm{mTBI}$, and PAP adherence in those with PTSD alone is poor $[94,95]$.

\section{Insomnia and Circadian Dysregulation}

Prolonged sleep latency and sleep fragmentation are common in both the acute ( $0-7$ days), subacute (7-90 days), and chronic phase ( $>90$ days) of mTBI. Insomnia is the most common sleep disorder following TBI of all severities, occurs in 30 $65 \%$ of those with chronic symptoms, and is more common with less severe and blast-related injuries $[39,76,96]$. Similar to hypersomnia, insomnia associated with $\mathrm{mTBI}$ is frequently confounded by other factors, particularly pain and comorbid psychiatric disorders. For example, mTBI is frequently associated with significant autonomic dysregulation and alterations in normal circadian patterns [53, 78]. Head injuries and neurotrauma may affect normal melatonin production [22]. Alterations in melatonin production and secretion following TBI not only both promote and propagate circadian dysregulation and insomnia, but it might also portend worse outcomes as melatonin provides neuroprotective properties in animal models [97].

Insomnia and alterations of the sleep-wake cycle that develop following mTBI are often complex and present treatment challenges, in part due to frequent physical injuries, comorbid PTSD, and other psychological conditions, the use of psychoactive medications, and alterations in melatonin production. Although sedative hypnotics such as benzodiazepines are commonly prescribed for this population, current TBI treatment guidelines recommend avoiding benzodiazepines if possible [10, 98], owing to risks for disinhibition, increased cognitive dysfunction, dependence, and other side effects. Nonbenzodiazepine receptor agonists such as zolpidem, zopiclone, and eszopiclone are also commonly prescribed, but there are no randomized studies assessing their safety or efficacy in patients with mTBI. Notably, a recent study reported that the use of hypnotics in patients with TBI of mixed severity was associated with an increased risk of dementia in this population [99].

CBTI is considered first-line treatment for chronic insomnia and can be successfully utilized in patients with mTBI [100-105]. Previous reports have shown that CBTI reduced daytime fatigue and improved nocturnal sleep quality among patients with TBI [48]. Similarly, a recent sleep health program by the Department of Veteran's Affairs, which incorporated CBTI, relaxation techniques, sleep restriction, stimulus control, and exercise proved beneficial in patients with persistent TBI symptoms [106]. Melatonin may serve a beneficial role in the treatment of mTBI-induced insomnia, but definitive trials are not yet available. Nonetheless, in light of the potential for alterations in melatonin production and secretion following mTBI, replacement therapy may improve sleep among these patients. However, in a study by Kemp et al. [107], melatonin did not improve sleep latency, duration, or quality among patients with TBI of mixed severity. In an observational study of patients with mixed-severity TBI and insomnia who received standard of care treatment for insomnia, focusing on CBTI, patients with improved sleep had decreased inflammatory markers as measured by $\mathrm{C}$-reactive protein [108]. Additional studies aimed at identifying the impact of insomnia on outcomes, exploring effective therapeutic options, and measuring the benefits that treating sleep disturbances has on neurorecovery are clearly needed. Importantly, identifying multiple strategies to treat adequately sleep and circadian disturbances in mTBI, using both existing and new pharmacological and cognitive-behavioral interventions, is required in order to determine which treatment(s) are most effective for an individual patient. Given the heterogeneity of MTBI and lack of operational consistency in the literature, clinical trials that can have a rapid impact and effectively improve practice parameters must be conducted within a framework of precision medicine. 


\section{What are Potentially Unique Mechanisms for Sleep Disturbance in mTBI?}

Although a detailed review is beyond the scope of this paper, multiple shared mechanisms that potentially underlie the relations between disturbed sleep and mTBI warrant mention here. For example, recent elucidation of the acute neurometabolic cascade after TBI and the cellular processes that may relate to both acute and chronic symptoms lends support to multiple levels of subtle damage to neurons, axons, microtubules, and other structures that can result in dysfunctional connections between critical links in the neural networks that regulate sleep drive, circadian rhythms, and sleep quality (i.e., sleep architecture) [109]. Similarly, acute changes that occur with brain injury impact cellular functions and neural microstructure, potentially causing cell death and more persistent changes in brain function, including increased sleep drive [12]. Other hypothesized shared mechanisms between sleep disturbance and TBI of varying severity, including mTBI, include impaired neurotransmitter function [54, 110-112], cerebrovascular autoregulatory dysfunction [113, 114], neuroinflammation [115], and dysregulation of circadian hormones $[22,116,117]$. In addition, interactions between mTBI and comorbid conditions such as PTSD [118], polytrauma, and chronic pain [119], have received increasing attention in the literature. These relationships are particularly salient as numerous studies have demonstrated heightened autonomic arousal in each of these conditions [120], likely increasing risk for adverse outcomes following mTBI. Finally, sleep disturbances are likely to contribute to longterm adverse effects following mTBI through impaired immune and degraded glymphatic function [121].

\section{What are Treatment Development Opportunities for Sleep Disturbances in mTBI?}

In light of the high prevalence of clinically significant sleep disturbances, as well as the potential neuroprotective and neurorecuperative effects of sleep, and the paucity of longitudinal and interventional studies in humans, it may be useful to divide the treatment strategies into phases temporally related to the injury. In the acute (or subacute) phase of injury, treatment and interventions should be aimed at mitigating the potential for long-term neurologic sequelae and minimizing the development of subsequent sleep disorders. Recent strategies developed in animal models have focused on altering the sleep-wake cycle. For example, $24 \mathrm{~h}$ of total sleep deprivation after TBI reduced the morphological damage and enhanced recovery in rats [37]. Another group confirmed that hippocampal neurogenesis was enhanced in rats as a result of short-term sleep deprivation following injury [38]. The effects of caffeine shortly following injury have also been studied. In a rat model, high-dose $(25 \mathrm{mg} / \mathrm{kg})$ caffeine administered within 1 min of injury blocked the downstream effects of an adenosine surge that is thought to mediate death due to prolonged apnea [122]. Although caffeine may prove to be advantageous in an animal model during the acute phase of injury, its benefits in humans are currently unknown. Indeed, it should be noted that $>30$ drugs that showed benefit in acute TBI animal models subsequently failed to show benefit in human trials, highlighting the challenging nature of translational research in this area. Indeed, in humans poor sleep following injury, particularly in the subacute phase, has been associated with worsened neuropsychiatric, behavioral, and somatic outcomes [123, 124]. Similarly, there is a substantial amount of data demonstrating the importance of adequate sleep following mTBI during the chronic phase of injury. Further, the Defense and Veterans Brain Injury Center warns against caffeine use during the chronic phase as it may worsen sleep fragmentation and insomnia [125]. Thus, alertnesspromoting strategies that manage and treat the daytime somnolence and fatigue that characterize mTBI and also minimally interfere with nocturnal sleep are greatly needed.

Beyond pharmacotherapy or sleep itself, researchers are increasingly interested in tools that promote neuromodulation. For example, noninvasive photic and auditory technologies have shown promising initial results in the chronic phase of treatment [126, 127]. The benefits of near-infrared light for intracellular healing have also been associated with reduced depressive severity and improved sleep quality over a 2month period among 10 patients with persistent TBI symptoms [128]. Perhaps the most exciting development in sleep research that has potential relevance for mTBI-related sleep disorders is the concept of glial lymphatics, or "glymphatics". Glymphatics is based on the observation that CSF flow differs during sleep and wakefulness, and improved CSF clearance can mediate neurotoxic proteins. For example, Xie et al. [21] reported increased clearance of interstitial waste during SWS in mice. These findings are pertinent not only for mTBI in general, but also for sleep disturbances in $\mathrm{mTBI}$, as there is increasing evidence that disrupted sleep can promote the accumulation of phosphorylated tau and $\beta$-amyloid proteins [129]. Modulation of this process may prove beneficial for the treatment of mTBI and other neurodegenerative disorders.

\section{What are Methodological Considerations in Studying Sleep in mTBI?}

Existing studies assessing sleep disturbances in patients with TBI have numerous limitations. Many are retrospective chart reviews utilizing clinical data and questionnaires that are not validated in patients with mTBI. As has been emphasized, preinjury sleep patterns or potentially undiagnosed sleep disorders are typically not assessed prior to injury, making it difficult to discern whether pre-existing sleep disorders might have contributed to the injury or development of chronic 
symptoms. Similarly, research evaluating insomnia is often limited to subjective data and rarely employs well-defined diagnostic or inclusion criteria. In addition, the comorbidity of psychiatric disorders and frequent use of psychoactive medications can lead to oversight of insomnia as an independent clinical disorder warranting treatment. Furthermore, few studies differentiate between the subjective complaints of insomnia symptoms, insomnia syndrome, sleep fragmentation due to OSA, and circadian rhythm disorders. In terms of TBI characteristics, most of the published research does not differentiate between mechanism, severity, or time since injury. Given these limitations, it is difficult to understand and appreciate the association of sleep disorders with mTBI and their potential impact on outcomes. The inclusion of representative samples in prospective studies and clinical trials is crucial if research findings are rapidly to impact patient care in an effective and personalized manner.

\section{Patient Selection}

Patients with mTBI suffer from a variety of sleep disturbances including insomnia (30-65\%) [118, 130], hypersomnia (1053\%) [89], and circadian rhythm abnormalities [53]. At the same time, mTBI is highly comorbid with psychiatric sequelae, including PTSD, anxiety, and mood disorders, as well as somatic complaints, including headaches, chronic pain, and fatigue. Further, mTBI is often associated with substance use disorders. Each of these comorbid conditions can worsen sleep. A central objective of future research must therefore be to elucidate the nature of sleep-wake disturbances in $\mathrm{mTBI}$, and it is crucial to reduce heterogeneity and to define subtypes of patients with TBI (i.e., patients without preexisting sleep, psychiatric, and substance disorders $v s$ those with these disorders) in clinical trials.

This scrutiny should be also extended to other factors that determine the nature of insult and its consequences following mTBI. They include, but are not limited to, postinjury time period, medication side effects, organic conditions with potential for interference (e.g., prior sleep disorders, thyroid malfunction, diabetes), and single versus multiple mTBI. The time interval since mTBI is of substantial importance because the sleep-wake disturbances following mTBI can start early and often persist for years after the trauma [86]. It is also important to evaluate the potential cumulative effects of repeated mTBI exposure on disease severity, and single versus repeated $\mathrm{mTBI}$ should be considered when designing studies to evaluate the associations between sleep and mTBI. In order to understand the natural course of sleep disturbances in patients with $\mathrm{mTBI}$, greater attention must be paid to variability in day-to-day (and night-to-night) symptoms among those experiencing sleep disturbances and mTBI. Finally, it will be essential to include control participants in longitudinal trial designs, including both matched healthy controls and individuals exposed to trauma but not concussed [86]. To ensure generalizable results, trial populations should be homogeneous with regard to the sleep disturbance itself. Characteristics of sleep disordered breathing (mild, moderate, or severe, as determined by objective testing), insomnia with or without comorbid disorders, hypersomnolence, and circadian rhythm disorders should be matched as closely as possible, especially for interventional studies. To meet these objectives and ensure real-world generalizability, large sample sizes will be required.

\section{Measurement Issues}

There appears to be a lack of concordance between subjective report, chart review, clinical assessment, and sleep laboratory measures in patients with mTBI. Therefore, multiple methods are essential to capture both subjective and objective measures of sleep, preferably with serial assessment. In addition to assessing central tendency, within-subject variability and night-to-night variability of sleep patterns must also be considered, as these are increasingly recognized as important determinants of disease severity and treatment outcome [131-134]. The utility of caregiver report should also be evaluated. Finally, there are reciprocal relationships between impaired daily function from mTBI, mood changes, chronic pain, and disrupted sleep at night. Experience sampling or ecological momentary assessments are likely to provide unique insights into the temporal nature of these relationships [135]. Further, each of these conditions amplifies sympathetic activation and exacerbates the other disorders. Hence, measurement of sympathetic tone during diagnostic sleep testing (e.g., heart rate variability, peripheral arterial tonometry, or pulse transit time) might be particularly helpful in objectively identifying sympathetic-parasympathetic imbalance and monitoring treatment outcomes. To advance patient-centered care, general and disease-specific HrQOL should be measured over time [136]. Finally, health economic measures, including direct and indirect costs, should be included as primary endpoints so that the most cost-effective treatments are identified [137].

\section{Clinical Trial Design}

Currently, there are no specific treatments for management of TBI-induced sleep disorders other than those used in patients without a TBI. Further, to date, most TBI treatment studies have produced disappointing results. In addition to the aforementioned heterogeneity and resulting risks for pseudo-specificity, studies to date have suffered from inconsistent measurement of sleep, as well as a lack of clearly defined and mTBI-specific endpoints or measures.

Given the complex physiological and cognitive-behavioral nature of sleep-wake disturbances after mTBI on the one hand, and absence of reliable outcome data on the other, it 
would be prudent to develop studies with multiple subjective and objective measures of sleep in the short (3-6 weeks), medium (3-4 months), and long term (6-12 months). It is essential to distinguish between managing sleep during the acute ( $0-7$ days) and subacute (7-90 days) phases and treating chronic sleep disorders following mTBI ( $>90$ days). Therefore, trial duration and endpoint measurement should also be consistent with the postconcussive period (acute, subacute, persistent postconcussive). Adaptive clinical trials are likely to be of particular benefit, as this approach will enable flexible attention paid to the variability of symptoms and facilitate a personalized medicine approach.

\section{A Roadmap for the Way Forward: Targeting Sleep Disturbances in mTBI}

In spite of over a decade of research and many millions of dollars of invested, clinical care for patients with mTBI too often produces disappointing results. Enhanced sleep and targeted sleep disorders treatments hold promise for rapidly improving outcomes and HrQOL among these patients. At the same time, scientific and practical challenges are evident. Our working group identified 3 pillars necessary to achieve the vision of improved outcomes in mTBI through improved sleep. Key recommendations are presented in Table 2. Perhaps most important, it is essential to recognize that a successful effort will require sustained commitment from multiple stakeholders, including academia, industry, governmental regulatory authorities, funding agencies, health systems, medical educators, and advocacy groups.

First, it is necessary to increase the quantity, quality, and efficiency of sleep-related data that are acquired during the many thousands of mTBI patient encounters each year. This necessitates capital investment in infrastructure. Establishment of a multisite data repository or expansion of data repositories already in existence, such as the Federal Interagency TBI Repository, to include greater amounts of sleep-related data is required. Such a repository will allow researchers to aggregate and share sleep-related data from multiple sites, employing validated measures, systematizing endpoints, and accelerating discovery. Such approaches are highly successful in other disease states (e.g., Alzheimer's dementia). Discussions are underway to determine the most efficient ways advance similar efforts among diverse stakeholders regarding sleep in mTBI.

Second, there is a clear need for improved understanding of the natural course of sleep disturbances in mTBI, including the neural, immune, endocrine, physiological, and cellular mechanisms underlying this relationship. In particular, improved understanding is required regarding the long-term consequences of single and multiple mTBI, including impact on neurodegeneration and HRQoL. This will help identify novel targets, so that new treatments can be developed. To achieve these objectives, multimethod and serial assessments of subjective and objective measures of sleep in mTBI are needed. Analysis of such longitudinal data will provide much-needed

Table 2 Key actionable recommendations to improve outcomes in sleep in patients with mild traumatic brain injury (mTBI)

\begin{tabular}{ll}
\hline Recommendation & Strategy and benefit \\
\hline $\begin{array}{l}\text { Scientific recommendations } \\
\text { Shared data repository }\end{array}$ & $\begin{array}{c}\text { Sleep-specific items should be incorporated into existing data repositories such as the Interagency Traumatic } \\
\text { Brain Injury Repository (FITBIR). In addition, a shared repository for detailed sleep measurement } \\
\text { (e.g., actigraphy, PSG, and neuroimaging) should be constructed and utilized by a core network } \\
\text { of sleep TBI clinical and research centers } \\
\text { Longitudinal studies should be conducted to understand changes in sleep over time in patients with mTBI } \\
\text { and to identify predictors of chronic sleep disorders following mTBI. These studies must employ } \\
\text { multimethod assessment of sleep and mTBI outcomes, including subjective and objective measures } \\
\text { and HRQoL. To increase speed, multisite studies can be conducted among a core network of sleep } \\
\text { TBI clinical and research centers } \\
\begin{array}{c}\text { Studies of targeted treatments for TBI-specific sleep disorders (e.g., post-traumatic hypersomnia/narcolepsy } \\
\text { and post-traumatic insomnia) are urgently needed. Standard treatments for sleep disorders require } \\
\text { optimization for mTBI. Particular emphasis should be put on psychiatric and other comorbidity, including } \\
\text { polytrauma. Economic measures must be included to enable identification the most cost-effective treatments }\end{array} \\
\begin{array}{c}\text { Improved treatment development } \\
\text { Improved provider education }\end{array} \\
\begin{array}{c}\text { A scalable sleep-specific educational curriculum including provider tools should be developed for head injury } \\
\text { medical professionals. A modular format should be employed for standalone use or incorporation into } \\
\text { existing curricula }\end{array} \\
\text { Dramatically increased access to sleep medicine services in head trauma centers is required. In addition to } \\
\text { improved provider education and greater collaboration within individual centers, opportunities to leverage } \\
\text { technology and incorporate tele-health should be pursued }\end{array}$ \\
\hline
\end{tabular}

$\mathrm{PSG}=$ polysomnography; HRQoL = health-related quality of life 
insight into both the natural course and neurorecuperative effects of sleep in patients with mTBI. Longitudinal analyses will also enable the identification of predictors of resilience to or development of chronic sleep disorders in TBI, and potentially predict other chronic postconcussive symptoms and even future risk for neurodegeneration. Because both sleep disturbances and mTBI negatively impact HRQoL, it will be especially fruitful to evaluate this relationship over time.

Finally and, most important, patients with mTBI require greater access to improved sleep treatments. This will require rapid development of targeted treatments for TBI-specific sleep disorders (e.g., post-traumatic hypersomnia/narcolepsy and post-traumatic insomnia), as well as adaptation and more effective dissemination of standard sleep treatments in patients with mTBI. In light of the frequent co-occurrence of mTBI and psychiatric disorders, adaptive clinical trials that target multiple symptoms are likely to be particular beneficial in refining current treatments to mTBI populations. In terms of increasing access to care, incorporating sleep-specific education into head injury curricula of health professionals and enabling access to sleep medicine services in head trauma centers are practical, feasible, and likely to provide rapid and substantial benefit. From a discovery perspective, development of future targeted therapies in sleep and mTBI is vital. These efforts will require consensus between practitioners, regulatory authorities, and pharmaceutical companies regarding the validation and acceptance of mTBI-related sleep disorders as appropriate indications for new drug development. In our view, and in each of these domains, partnerships between clinicians, regulatory authorities and other governmental agencies, and interested pharmaceutical companies are essential.

Required Author Forms Disclosure forms provided by the authors are available with the online version of this article.

\section{Compliance with Ethical Standards}

Disclaimer The views, opinions, and/or findings contained in this report are those of the authors and should not be construed as an official Department of Defense or Veterans Affairs position, policy or decision unless so designated by other documentation.

\section{References}

1. Faul M, Xu L, Wald MM, Coronado V. Traumatic brain injury in the United States. Atlanta, GA: Centers for Disease Control and Prevention, National Center for Injury Prevention and Control 2010.

2. Leo P, McCrea M. Epidemiology. In: Laskowitz D, Grant G, editors. Translational research in traumatic brain injury. Boca Raton (FL): by Taylor \& Francis Group, LLC; 2016.
3. National Center for Injury Prevention and Control. Report to Congress on Mild Traumatic Brain Injury in the United States: Steps to prevent a serious public health problem. 2003.

4. Corrigan JD, Selassie AW, Orman JA. The epidemiology of traumatic brain injury. J Head Trauma Rehabil 2010;25:72-80.

5. Gerberding J. for the National Center for Injury Prevention and Control. Report to congress on mild traumatic brain injury in the United States - steps to prevent a serious public health problem. 2003.

6. Mathias JL, Alvaro PK. Prevalence of sleep disturbances, disorders, and problems following traumatic brain injury: a meta-analysis. Sleep Med 2012;13:898-905.

7. Kay T, Harrington D, Adams R. American Congress of Rehabilitation Medicine, Head Injury Interdisciplinary Special Interest Group. Definition of mild traumatic brain injury. J Head Trauma Rehabil 1993;8:86-87.

8. Menon DK, Schwab K, Wright DW, Maas AI, Demographics and Clinical Assessment Working Group of the International and Interagency Initiative toward Common Data Elements for Research on Traumatic Brain Injury and Psychological Health. Position statement: definition of traumatic brain injury. Arch Phys Med Rehabil 2010;91:1637-1640.

9. Teasdale G, Jennett B. Assessment of coma and impaired consciousness: a practical scale. Lancet 1974;304:81-84.

10. Management of Concussion/mTBI Working Group. VA/DoD Clinical Practice Guideline for Management of Concussion/Mild Traumatic Brain Injury. J Rehabil Res Dev 2009;46:CP1-68.

11. Amyot F, Arciniegas DB, Brazaitis MP, et al. A review of the effectiveness of neuroimaging modalities for the detection of traumatic brain injury. J Neurotrauma 2015;32:1693-1721.

12. Giza CC, Hovda DA. The new neurometabolic cascade of concussion. Neurosurgery 2014;75(Suppl. 4):S24-S33.

13. Centers for Disease control and Prevention. What are the signs and symptoms of concussion. Available at: http://www.cdc.gov/ traumaticbraininjury/symptoms.html. Accessed October 1, 2015.

14. Jaffee MS, Winter WC, Jones CC, Ling G. Sleep disturbances in athletic concussion. Brain Inj 2015;29:221-227.

15. Assefa SZ, Diaz-Abad M, Wickwire EM, Scharf SM. The functions of sleep. AIMS Neuroscience 2015;2(3):155-171.

16. Berry R, Brooks R, Gamaldo C, Harding S, Marcus C, Vaughn B. The AASM manual for the scoring of sleep and associated events: rules, terminology and technical specifications, version 2.0. Darien, Illinois: American Academy of Sleep Medicine 2012:47.

17. Saper CB, Fuller PM, Pedersen NP, Lu J, Scammell TE. Sleep state switching. Neuron 2010;68:1023-1042.

18. Van Dongen HP, Maislin G, Mullington JM, Dinges DF. The cumulative cost of additional wakefulness: dose-response effects on neurobehavioral functions and sleep physiology from chronic sleep restriction and total sleep deprivation. Sleep 2003;26:117126.

19. Banks S, Dinges DF. Behavioral and physiological consequences of sleep restriction. J Clin Sleep Med 2007;3:519-528.

20. Cirelli C. Brain plasticity, sleep and aging. Gerontology 2012;58: 441-445.

21. Xie L, Kang H, Xu Q, et al. Sleep drives metabolite clearance from the adult brain. Science 2013;342:373-377.

22. Shekleton JA, Parcell DL, Redman JR, Phipps-Nelson J, Ponsford JL, Rajaratnam SM. Sleep disturbance and melatonin levels following traumatic brain injury. Neurology 2010;74:1732-1738.

23. Weil ZM, Norman GJ, Karelina K, et al. Sleep deprivation attenuates inflammatory responses and ischemic cell death. Exp Neurol 2009;218:129-136.

24. Hsu JC, Lee YS, Chang CN, Ling EA, Lan CT. Sleep deprivation prior to transient global cerebral ischemia attenuates glial reaction in the rat hippocampal formation. Brain Res 2003;984:170-181. 
25. Moldovan M, Constantinescu AO, Balseanu A, Oprescu N, Zagrean L, Popa-Wagner A. Sleep deprivation attenuates experimental stroke severity in rats. Exp Neurol 2010;222:135-143.

26. Dirnagl U, Becker K, Meisel A. Preconditioning and tolerance against cerebral ischaemia: from experimental strategies to clinical use. Lancet Neurol 2009;8:398-412.

27. Pace M, Baracchi F, Gao B, Bassetti C. Identification of sleepmodulated pathways involved in neuroprotection from stroke. Sleep 2015;38:1707-1718.

28. Cam E, Gao B, Imbach L, Hodor A, Bassetti CL. Sleep deprivation before stroke is neuroprotective: a pre-ischemic conditioning related to sleep rebound. Exp Neurol 2013;247:673-679.

29. Gao B, Cam E, Jaeger H, Zunzunegui C, Sarnthein J, Bassetti CL. Sleep disruption aggravates focal cerebral ischemia in the rat. Sleep 2010;33:879-887.

30. Zunzunegui C, Gao B, Cam E, Hodor A, Bassetti CL. Sleep disturbance impairs stroke recovery in the rat. Sleep 2011;34:12611269.

31. Franken P, Dijk DJ, Tobler I, Borbely AA. Sleep deprivation in rats: effects on EEG power spectra, vigilance states, and cortical temperature. Am J Physiol 1991;261:R198-208.

32. Dash MB, Douglas CL, Vyazovskiy VV, Cirelli C, Tononi G. Long-term homeostasis of extracellular glutamate in the rat cerebral cortex across sleep and waking states. J Neurosci 2009;29: 620-629.

33. Kong J, Shepel PN, Holden CP, Mackiewicz M, Pack AI, Geiger JD. Brain glycogen decreases with increased periods of wakefulness: implications for homeostatic drive to sleep. J Neurosci 2002;22:5581-5587.

34. Shearer WT, Reuben JM, Mullington JM, et al. Soluble TNFalpha receptor 1 and IL-6 plasma levels in humans subjected to the sleep deprivation model of spaceflight. J Allergy Clin Immunol 2001;107:165-170.

35. Vgontzas AN, Zoumakis E, Bixler EO, et al. Adverse effects of modest sleep restriction on sleepiness, performance, and inflammatory cytokines. J Clin Endocrinol Metab 2004;89:2119-2126.

36. Hodor A, Palchykova S, Baracchi F, Noain D, Bassetti CL. Baclofen facilitates sleep, neuroplasticity, and recovery after stroke in rats. Ann Clin Transl Neurol 2014;1:765-777.

37. Martinez-Vargas M, Estrada Rojo F, Tabla-Ramon E, et al. Sleep deprivation has a neuroprotective role in a traumatic brain injury of the rat. Neurosci Lett 2012;529:118-122.

38. Cheng O, Li R, Zhao L, et al. Short-term sleep deprivation stimulates hippocampal neurogenesis in rats following global cerebral ischemia/reperfusion. PLoS One 2015;10:e0125877.

39. Ouellet MC, Beaulieu-Bonneau S, Morin CM. Insomnia in patients with traumatic brain injury: frequency, characteristics, and risk factors. J Head Trauma Rehabil 2006;21:199-212.

40. Gagner C, Landry-Roy C, Laine F, Beauchamp MH. Sleep-wake disturbances and fatigue after pediatric traumatic brain injury: a systematic review of the literature. J Neurotrauma 2015;32:15391552.

41. Tham SW, Fales J, Palermo TM. Subjective and objective assessment of sleep in adolescents with mild traumatic brain injury. J Neurotrauma 2015;32:847-852.

42. Buysse DJ, Reynolds CF,3rd, Monk TH, Berman SR, Kupfer DJ. The Pittsburgh Sleep Quality Index: a new instrument for psychiatric practice and research. Psychiatry Res 1989;28:193-213.

43. Johns MW. A new method for measuring daytime sleepiness: the Epworth sleepiness scale. Sleep 1991;14:540-545.

44. Masel BE, Scheibel RS, Kimbark T, Kuna ST. Excessive daytime sleepiness in adults with brain injuries. Arch Phys Med Rehabil 2001;82:1526-1532.

45. Lequerica A, Chiaravalloti N, Cantor J, et al. The factor structure of the Pittsburgh Sleep Quality Index in persons with traumatic brain injury. A NIDRR TBI model systems module study. NeuroRehabilitation 2014;35:485-492.

46. Fichtenberg NL, Putnam SH, Mann NR, Zafonte RD, Millard AE. Insomnia screening in postacute traumatic brain injury: utility and validity of the Pittsburgh Sleep Quality Index. Am J Phys Med Rehabil 2001;80:339-345.

47. Parcell DL, Ponsford JL, Rajaratnam SM, Redman JR. Selfreported changes to nighttime sleep after traumatic brain injury. Arch Phys Med Rehabil 2006;87:278-285.

48. Ouellet MC, Morin CM. Efficacy of cognitive-behavioral therapy for insomnia associated with traumatic brain injury: a single-case experimental design. Arch Phys Med Rehabil 2007;88:15811592.

49. Buysse DJ, Ancoli-lsrael S, Edinger JD, Lichstein KL, Morin CM. Recommendations for a standard research assessment of insomnia. Sleep 2006;29:1155-1173.

50. Lam JC, Mahone EM, Mason TB, Scharf SM. Defining the roles of actigraphy and parent logs for assessing sleep variables in preschool children. Behav Sleep Med 2011;9:184-193.

51. Zollman FS, Cyborski C, Duraski SA. Actigraphy for assessment of sleep in traumatic brain injury: case series, review of the literature and proposed criteria for use. Brain Inj 2010;24:748-754.

52. Ancoli-Israel S, Cole R, Alessi C, Chambers M, Moorcroft W, Pollak CP. The role of actigraphy in the study of sleep and circadian rhythms. Sleep 2003;26:342-392.

53. Ayalon L, Borodkin K, Dishon L, Kanety H, Dagan Y. Circadian rhythm sleep disorders following mild traumatic brain injury. Neurology 2007;68:1136-1140.

54. Baumann CR, Bassetti CL, Valko PO, et al. Loss of hypocretin (orexin) neurons with traumatic brain injury. Ann Neurol 2009;66: 555-559.

55. Makley MJ, Johnson-Greene L, Tarwater PM, et al. Return of memory and sleep efficiency following moderate to severe closed head injury. Neurorehabil Neural Repair 2009;23:320-326.

56. Chiu HY, Lo WC, Chiang YH, Tsai PS. The effects of sleep on the relationship between brain injury severity and recovery of cognitive function: a prospective study. Int J Nurs Stud 2014;51:892899.

57. Sinclair KL, Ponsford J, Rajaratnam SM. Actigraphic assessment of sleep disturbances following traumatic brain injury. Behav Sleep Med 2014;12:13-27.

58. Lequerica A, Jasey N, Portelli Tremont JN, Chiaravalloti ND. Pilot study on the effect of ramelteon on sleep disturbance after traumatic brain injury: preliminary evidence from a clinical trial. Arch Phys Med Rehabil 2015;96:1802-1809.

59. Laakso ML, Leinonen L, Lindblom N, Joutsiniemi SL, Kaski M. Wrist actigraphy in estimation of sleep and wake in intellectually disabled subjects with motor handicaps. Sleep Med 2004;5:541550.

60. Rao V, Bergey A, Hill H, Efron D, McCann U. Sleep disturbance after mild traumatic brain injury: indicator of injury? J Neuropsychiatry Clin Neurosci 2011;23:201-205.

61. Khoury S, Chouchou F, Amzica F, et al. Rapid EEG activity during sleep dominates in mild traumatic brain injury patients with acute pain. J Neurotrauma 2013;30:633-641.

62. Newell J, Mairesse O, Verbanck P, Neu D. Is a one-night stay in the lab really enough to conclude? First-night effect and night-tonight variability in polysomnographic recordings among different clinical population samples. Psychiatry Res 2012;200:795-801.

63. Lu W, Cantor J, Aurora RN, et al. Variability of respiration and sleep during polysomnography in individuals with TBI. NeuroRehabilitation 2014;35:245-251.

64. Plante DT, Goldstein MR, Cook JD, et al. Effects of partial sleep deprivation on slow waves during non-rapid eye movement sleep: 
a high density EEG investigation. Clin Neurophysiol 2016;127: 1436-1444.

65. Poryazova R, Huber R, Khatami R, et al. Topographic sleep EEG changes in the acute and chronic stage of hemispheric stroke. $\mathrm{J}$ Sleep Res 2015;24:54-65.

66. Nofzinger EA. Neuroimaging and sleep medicine. Sleep Med Rev 2005;9:157-172.

67. Pisarenco I, Caporro M, Prosperetti C, Manconi M. High-density electroencephalography as an innovative tool to explore sleep physiology and sleep related disorders. Int J Psychophysiol 2014;pii:S0167-8760(14)00003-8.

68. Hasler BP, Insana SP, James JA, Germain A. Evening-type military veterans report worse lifetime posttraumatic stress symptoms and greater brainstem activity across wakefulness and REM sleep. Biol Psychol 2013;94:255-262.

69. Germain A, James J, Insana S, et al. A window into the invisible wound of war: functional neuroimaging of REM sleep in returning combat veterans with PTSD. Psychiatry Res 2013;211:176-179.

70. Peskind ER, Petrie EC, Cross DJ, et al. Cerebrocerebellar hypometabolism associated with repetitive blast exposure mild traumatic brain injury in 12 Iraq war Veterans with persistent post-concussive symptoms. Neuroimage 2011;54(Suppl. 1):S76S82.

71. Stocker RP, Cieply MA, Paul B, et al. Combat-related blast exposure and traumatic brain injury influence brain glucose metabolism during REM sleep in military veterans. Neuroimage 2014;99: 207-214.

72. Delouche A, Attye A, Heck O, et al. Diffusion MRI: Pitfalls, literature review and future directions of research in mild traumatic brain injury. Eur J Radiol 2016;85:25-30.

73. Currie S, Saleem N, Straiton JA, Macmullen-Price J, Warren DJ, Craven IJ. Imaging assessment of traumatic brain injury. Postgrad Med J 2016;92:41-50.

74. Bruce ED, Konda S, Dean DD, Wang EW, Huang JH, Little DM. Neuroimaging and traumatic brain injury: State of the field and voids in translational knowledge. Mol Cell Neurosci 2015;66:103113

75. Chong CD, Schwedt TJ. White matter damage and brain network alterations in concussed patients: a review of recent diffusion tensor imaging and resting-state functional connectivity data. Curr Pain Headache Rep 2015;19:485-015-0485-0.

76. Collen J, Orr N, Lettieri CJ, Carter K, Holley AB. Sleep disturbances among soldiers with combat-related traumatic brain injury. Chest 2012;142:622-630.

77. Verma A, Anand V, Verma NP. Sleep disorders in chronic traumatic brain injury. J Clin Sleep Med 2007;3:357-362.

78. Kempf J, Werth E, Kaiser PR, Bassetti CL, Baumann CR. Sleepwake disturbances 3 years after traumatic brain injury. J Neurol Neurosurg Psychiatry 2010;81:1402-1405.

79. Ouellet MC, Morin CM. Subjective and objective measures of insomnia in the context of traumatic brain injury: a preliminary study. Sleep Med 2006;7:486-497.

80. Castriotta RJ, Wilde MC, Lai JM, Atanasov S, Masel BE, Kuna ST. Prevalence and consequences of sleep disorders in traumatic brain injury. J Clin Sleep Med 2007;3:349-356.

81. Parcell DL, Ponsford JL, Redman JR, Rajaratnam SM. Poor sleep quality and changes in objectively recorded sleep after traumatic brain injury: a preliminary study. Arch Phys Med Rehabil 2008;89:843-850.

82. Sommerauer M, Valko PO, Werth E, Baumann CR. Excessive sleep need following traumatic brain injury: a case-control study of 36 patients. J Sleep Res 2013;22:634-639.

83. Cote KA, Milner CE, Speth TA. Altered sleep mechanisms following traumatic brain injury and relation to waking function. AIMS Neuroscience 2015;2(4):203-228.
84. Schreiber S, Barkai G, Gur-Hartman T, et al. Long-lasting sleep patterns of adult patients with minor traumatic brain injury (mTBI) and non-mTBI subjects. Sleep Med 2008;9:481-487.

85. Williams BR, Lazic SE, Ogilvie RD. Polysomnographic and quantitative EEG analysis of subjects with long-term insomnia complaints associated with mild traumatic brain injury. Clin Neurophysiol 2008;119:429-438.

86. Ouellet MC, Beaulieu-Bonneau S, Morin CM. Sleep-wake disturbances after traumatic brain injury. Lancet Neurol 2015;14(7): 746-757.

87. Baumann CR, Werth E, Stocker R, Ludwig S, Bassetti CL. Sleepwake disturbances 6 months after traumatic brain injury: a prospective study. Brain 2007;130:1873-1883.

88. Kaiser PR, Valko PO, Werth E, et al. Modafinil ameliorates excessive daytime sleepiness after traumatic brain injury. Neurology 2010;75:1780-1785.

89. Menn SJ, Yang R, Lankford A. Armodafinil for the treatment of excessive sleepiness associated with mild or moderate closed traumatic brain injury: a 12-week, randomized, double-blind study followed by a 12-month open-label extension. J Clin Sleep Med 2014;10:1181-1191.

90. Ponsford JL, Ziino C, Parcell DL, et al. Fatigue and sleep disturbance following traumatic brain injury-their nature, causes, and potential treatments. J Head Trauma Rehabil 2012;27:224-233.

91. Wilde MC, Castriotta RJ, Lai JM, Atanasov S, Masel BE, Kuna ST. Cognitive impairment in patients with traumatic brain injury and obstructive sleep apnea. Arch Phys Med Rehabil 2007;88: 1284-1288.

92. Wickwire EM, Lettieri CJ, Cairns AA, Collop NA. Maximizing positive airway pressure adherence in adults: a common-sense approach. Chest 2013;144:680-693.

93. Wickwire EM, Smith MT, Birnbaum S, Collop NA. Sleep maintenance insomnia complaints predict poor CPAP adherence: a clinical case series. Sleep Med 2010;11:772-776.

94. Collen JF, Lettieri CJ, Hoffman M. The impact of posttraumatic stress disorder on CPAP adherence in patients with obstructive sleep apnea. J Clin Sleep Med 2012;8:667-672.

95. Lettieri CJ, Williams SG, Collen JF. Obstructive sleep apnea syndrome and post-traumatic stress disorder: Clinical outcomes and impact of PAP therapy. Chest 2015 Aug 20 [Epub ahead of print].

96. Clinchot DM, Bogner J, Mysiw WJ, Fugate L, Corrigan J. Defining sleep disturbance after brain injury. Am J Phys Med Rehabil 1998;77:291-295.

97. Ates O, Cayli S, Gurses I, et al. Effect of pinealectomy and melatonin replacement on morphological and biochemical recovery after traumatic brain injury. Int J Dev Neurosci 2006;24:357-363.

98. Colorado Division of Workers' Compensation. Traumatic brain injury medical treatment guidelines. 2012:1-119.

99. Chiu HY, Lin EY, Wei L, et al. Hypnotics use but not insomnia increased the risk of dementia in traumatic brain injury patients. Eur Neuropsychopharmacol 2015;25:2271-2277.

100. Morin CM, Colecchi C, Stone J, Sood R, Brink D. Behavioral and pharmacological therapies for late-life insomnia: a randomized controlled trial. JAMA 1999;281:991-999.

101. Jacobs GD, Pace-Schott EF, Stickgold R, Otto MW. Cognitive behavior therapy and pharmacotherapy for insomnia: a randomized controlled trial and direct comparison. Arch Intern Med 2004; 164:1888-1896.

102. Sivertsen B, Omvik S, Pallesen S, et al. Cognitive behavioral therapy vs zopiclone for treatment of chronic primary insomnia in older adults: a randomized controlled trial. JAMA 2006;295: 2851-2858.

103. Morin CM, Vallieres A, Guay B, et al. Cognitive behavioral therapy, singly and combined with medication, for persistent insomnia: a randomized controlled trial. JAMA 2009;301:2005-2015. 
104. No authors listed. NIH. State of the science conference statement on manifestations and management of chronic insomnia in adults statement. J Clin Sleep Med 2005;1:412-421.

105. Schutte-Rodin S, Broch L, Buysse D, Dorsey C, Sateia M. Clinical guideline for the evaluation and management of chronic insomnia in adults. J Clin Sleep Med 2008;4:487-504.

106. De La Rue-Evans L, Nesbitt K, Oka RK. Sleep hygiene program implementation in patients with traumatic brain injury. Rehabil Nurs 2013;38:2-10.

107. Kemp S, Biswas R, Neumann V, Coughlan A. The value of melatonin for sleep disorders occurring post-head injury: a pilot RCT. Brain Inj 2004;18:911-919.

108. Barr T, Livingston W, Guardado P, Baxter T, Mysliwiec V, Gill J. Chapter 8 military personnel with traumatic brain injuries and insomnia have reductions in PTSD and improved perceived health following sleep restoration: a relationship moderated by inflammation. Annu Rev Nurs Res 2015;33:249-266.

109. Dean PJ, Sato JR, Vieira G, McNamara A, Sterr A. Long-term structural changes after mTBI and their relation to post-concussion symptoms. Brain Inj 2015 Jun 11 [Epub ahead of print].

110. Valko PO, Gavrilov YV, Yamamoto M, et al. Damage to histaminergic tuberomammillary neurons and other hypothalamic neurons with traumatic brain injury. Ann Neurol 2015;77:177-182.

111. Baumann CR, Stocker R, Imhof HG, et al. Hypocretin-1 (orexin A) deficiency in acute traumatic brain injury. Neurology 2005;65: 147-149.

112. Rye DB, Bliwise DL, Parker K, et al. Modulation of vigilance in the primary hypersomnias by endogenous enhancement of GABAA receptors. Sci Transl Med 2012;4:161ra151.

113. Becelewski J, Pierzchala K. Cerebrovascular reactivity in patients with mild head injury. Neurol Neurochir Pol 2003;37:339-350.

114. Junger EC, Newell DW, Grant GA, et al. Cerebral autoregulation following minor head injury. J Neurosurg 1997;86:425-432.

115. Kumar A, Loane DJ. Neuroinflammation after traumatic brain injury: opportunities for therapeutic intervention. Brain Behav Immun 2012;26:1191-1201.

116. Paparrigopoulos T, Melissaki A, Tsekou H, et al. Melatonin secretion after head injury: a pilot study. Brain Inj 2006;20:873-878.

117. Seifman MA, Gomes K, Nguyen PN, et al. Measurement of serum melatonin in intensive care unit patients: changes in traumatic brain injury, trauma, and medical conditions. Front Neurol 2014;5:237.

118. Gilbert KS, Kark SM, Gehrman P, Bogdanova Y. Sleep disturbances, TBI and PTSD: Implications for treatment and recovery. Clin Psychol Rev 2015;40:195-212.

119. Lavigne G, Khoury S, Chauny JM, Desautels A. Pain and sleep in post-concussion/mild traumatic brain injury. Pain 2015;156(Suppl. 1):S75-S85.

120. Tan G, Fink B, Dao TK, et al. Associations among pain, PTSD, $\mathrm{mTBI}$, and heart rate variability in veterans of Operation Enduring and Iraqi Freedom: a pilot study. Pain Med 2009;10:1237-1245.
121. Blaylock RL, Maroon J. Immunoexcitotoxicity as a central mechanism in chronic traumatic encephalopathy: a unifying hypothesis. Surg Neurol Int 2011;2:107.

122. Lusardi TA, Lytle NK, Szybala C, Boison D. Caffeine prevents acute mortality after TBI in rats without increased morbidity. Exp Neurol 2012;234:161-168.

123. Terrio H, Brenner LA, Ivins BJ, et al. Traumatic brain injury screening: preliminary findings in a US Army Brigade Combat Team. J Head Trauma Rehabil 2009;24:14-23.

124. Farrell-Carnahan L, Franke L, Graham C, McNamee S. Subjective sleep disturbance in veterans receiving care in the Veterans Affairs Polytrauma System following blast-related mild traumatic brain injury. Mil Med 2013;178:951-956.

125. Defense Veterans Brain Injury Center. TBI basics. Available at: http://dvbic.dcoe.mil/about-traumatic-brain-injury/article/tbibasics. Accessed October 1, 2015.

126. Gerdes L, Gerdes P, Lee SW, H Tegeler C. HIRREM: a noninvasive, allostatic methodology for relaxation and auto-calibration of neural oscillations. Brain Behav 2013;3:193-205.

127. Lee SW, Gerdes L, Tegeler CL, Shaltout HA, Tegeler CH. A bihemispheric autonomic model for traumatic stress effects on health and behavior. Front Psychol 2014;5:843.

128. Morries LD, Cassano P, Henderson TA. Treatments for traumatic brain injury with emphasis on transcranial near-infrared laser phototherapy. Neuropsychiatr Dis Treat 2015;11:2159-2175.

129. Lucke-Wold BP, Smith KE, Nguyen L, et al. Sleep disruption and the sequelae associated with traumatic brain injury. Neurosci Biobehav Rev 2015;55:68-77.

130. Dikmen S, McLean A, Temkin N. Neuropsychological and psychosocial consequences of minor head injury. J Neurol Neurosurg Psychiatry 1986;49:1227-1232.

131. Suh S, Nowakowski S, Bernert RA, et al. Clinical significance of night-to-night sleep variability in insomnia. Sleep Med 2012;13: 469-475.

132. Okun ML, Reynolds CF, 3rd, Buysse DJ, et al. Sleep variability, health-related practices, and inflammatory markers in a community dwelling sample of older adults. Psychosom Med 2011;73:142150.

133. Buysse DJ, Cheng Y, Germain A, et al. Night-to-night sleep variability in older adults with and without chronic insomnia. Sleep Med 2010;11:56-64.

134. Sanchez-Ortuno MM, Edinger JD. Internight sleep variability: its clinical significance and responsiveness to treatment in primary and comorbid insomnia. J Sleep Res 2012;21:527-534.

135. Shiffman S, Stone AA, Hufford MR. Ecological momentary assessment. Annu Rev Clin Psychol 2008;4:1-32.

136. Polinder S, Haagsma JA, van Klaveren D, Steyerberg EW, van Beeck EF. Health-related quality of life after TBI: a systematic review of study design, instruments, measurement properties, and outcome. Popul Health Metr 2015;13:4.

137. Wickwire EM, Shaya FT, Scharf SM. Health economics of insomnia treatments: The return on investment for a good night's sleep. Sleep Medicine Reviews 30(2016):72-82. 